\title{
Are acute sitting-induced changes in inflammation and cerebrovascular function related to impaired mood and cognition?
}

\author{
Sophie E. Carter ${ }^{1,2}$ (D) Richard Draijer ${ }^{3} \cdot$ Claire E. Stewart $^{1}$ - Andy D. Moss ${ }^{1}$ - Dick H. J. Thijssen ${ }^{1,4} \cdot$ Nicola D. Hopkins ${ }^{1}$
}

Received: 11 September 2020 / Accepted: 27 March 2021 / Published online: 19 April 2021

(c) The Author(s) 2021

\begin{abstract}
Purpose Sedentary behaviour is negatively associated with mood and cognition, yet how acute sitting contributes to these overall associations is unknown. Since sitting heightens inflammation and impairs cerebrovascular function, this study investigated the hypothesis that these sitting-induced changes are related to impaired mood and cognition.

Methods Twenty-five healthy desk workers (18 male, $28.3 \pm 7.5$ years, BMI: $24.2 \pm 3.3 \mathrm{~kg} \cdot \mathrm{m}^{-2}$ ) were recruited. During laboratory visit one, participants were familiarised with cognitive performance tests measuring executive function, attention and working memory. During laboratory visit two, participants completed $6 \mathrm{~h}$ of continuous, uninterrupted sitting. At baseline and after $6 \mathrm{~h}$, serum markers of inflammation, middle cerebral artery blood flow velocity (MCAv), cerebrovascular carbon dioxide reactivity (CVR), dynamic cerebral autoregulation (CA), cognitive performance and mood (positive and negative affect, alert, contented and calm) were assessed. Data were analysed using paired-samples t tests and correlation analyses. Results Following sitting, C-reactive protein $(\Delta-1.0 \mu \mathrm{g} / \mathrm{ml})$ and tissue plasminogen activator $(\Delta-360.4 \mathrm{pg} / \mathrm{ml})$ decreased $(p<0.05)$, MCAv reduced $\left(\Delta-2.9 \mathrm{~cm} \bullet \mathrm{s}^{-1}, p=0.012\right)$ and normalised gain increased in the very low frequency range, indicating impaired CA $\left(\Delta+0.22 \% \cdot \mathrm{mmHg}^{-1}, p=0.016\right)$. Positive affect $(\Delta-4.6, p<0.001)$, and alert $(\Delta-10.6 p=0.002)$ and contented $(\Delta-7.4, p=0.006)$ mood states also decreased following sitting. No significant changes in interleukin- 6 , tumour necrosis factor-alpha, von Willebrand factor, CVR or cognitive performance were observed $(p>0.05)$. The observed changes in inflammation and cerebrovascular function were not related to changes in mood $(p>0.05)$.

Conclusion Alterations in inflammation or cerebrovascular function following six hours of prolonged, uninterrupted sitting are not related to the observed reductions in mood, indicating other mechanisms underlie the relationship between acute sitting and mood disturbances.
\end{abstract}

Keywords Sedentary behaviour $\cdot$ Sitting $\cdot$ Cerebral blood flow $\cdot$ Cerebral autoregulation

\begin{tabular}{lll}
$\begin{array}{l}\text { Abbreviations } \\
\mathrm{BP} \quad \text { Blood pressure }\end{array}$ & CA & Cerebral autoregulation \\
$\mathrm{CO}_{2} \quad$ Carbon dioxide & CBF & Cerebral blood flow \\
& CVR & Cerebrovascular carbon dioxide reactivity \\
\hline $\begin{array}{l}\text { Sophie E. Carter } \\
\text { s.carter@yorksj.ac.uk }\end{array}$ & CVC & Cerebrovascular conductance \\
& CCA & Common carotid artery \\
Research Institute for Sport and Exercise Sciences, & HR & Heart rate \\
Liverpool John Moores University, Liverpool, UK & HF & High frequency \\
2 School of Science, Technology and Health, York St John & hs-CRP & High-sensitivity c-reactive protein \\
University, Nestlé Rowntree Park Sports Campus, Haxby & IL-6 & Interleukin-6 \\
Road, York YO31 8TA, UK & LF & Low frequency \\
Unilever Foods Innovation Centre, Wageningen, & MAP & Mean arterial pressure \\
The Netherlands & MCAv & Middle cerebral artery blood flow velocity \\
Radboud Institute for Health Sciences, Department & PANAS & Positive and negative affect schedule \\
of Physiology, Radboud University Medical Center, & PETCO & Pressure of end tidal CO ${ }_{2}$ \\
Nijmegen, The Netherlands & SB & Sedentary behaviour
\end{tabular}


TNF- $\alpha \quad$ Tumour necrosis factor-alpha

VLF Very low frequency

vWv von Willebrand factor

\section{Introduction}

High amounts of sedentary behaviour (SB), any waking behaviour in a sitting, reclining or lying posture [1], are associated with clinical mood disorders, such as depression [2] and anxiety [3], and decreased cognition [4, 5]. Acute increase in sitting also negatively affects mood $[6,7]$ and cognition $[8,9]$. Owing to the high contribution sitting has on total adult SB [10], understanding how acute sitting episodes contribute to overall associations between SB and mood and cognition is required. One potential mechanism may relate to increased inflammation. Inflammation is a critical mediator in the pathophysiology of mood disorders [11]. Acute elevation in inflammatory markers increases negative mood [12] and prolonged sitting acutely increases salivary interleukin(IL)-8 levels [13]. Furthermore, following two weeks of increased free-living SB, individuals with greater mood disturbance had an elevated IL-6 response to a stress test [14]. Sitting may therefore contribute to negative mood by enhancing inflammatory responses, but this has not been explored.

Impairments in cerebrovascular function, the regulatory mechanisms that maintain cerebral blood flow (CBF) [15], may also contribute to the associations between SB and cognition. Long-term impairment of cerebrovascular function is implicit in neurodegenerative diseases [16] and cognitive impairment [16]. Acute, prolonged sitting reduces CBF [17] and acute changes in CBF may influence cognition [18]. However, whether acute sitting-induced changes in cerebrovascular function are related to acute decrements in cognition is unknown. This study therefore investigated the relationship between sitting-induced alterations in inflammation and cerebrovascular function and changes in mood and cognition. We hypothesised that prolonged, uninterrupted sitting would increase inflammation and impair cerebrovascular function, and that these changes would be related to acute impairments in mood and cognition.

\section{Methods}

\section{Participants}

Twenty-five healthy desk workers (18 male) volunteered. Participants were screened prior to testing for exclusion criteria including: use of medication, smoker, BMI $>35$ or $<18 \mathrm{~kg} \cdot \mathrm{m}^{-2}$, use of hormone-based contraception and diagnosis of cerebrovascular, cardiovascular or metabolic disease. Written informed consent was obtained prior to inclusion.

\section{Study design}

Study procedures were approved by the Liverpool John Moores University Ethics Committee (16/SPS/031) and conformed to the Declaration of Helsinki. Participants attended the laboratory on two occasions: (1) familiarisation visit of the cognitive performance tests; (2) experimental visit which occurred the following day. For the experimental visit, participants arrived at the laboratory between 7.00 and 9.00 am and, following a 20-min supine rest, baseline supine middle cerebral artery blood flow velocity (MCAv) and cerebrovascular carbon dioxide reactivity (CVR) were assessed. Participants were then seated and underwent measures of seated MCAv and cerebral autoregulation (CA), and a venous blood sample was collected. Following this, the same cognitive performance tests as in the familiarisation visit were undertaken and two mood questionnaires completed. After these tests (PRE), participants completed a $6 \mathrm{~h}$ continuous, uninterrupted sitting period. This sitting duration was based on previous research which observed reduced mood following $6 \mathrm{~h}$ of uninterrupted sitting [6]. The measurement of seated MCAv was repeated immediately after the $6 \mathrm{~h}$ intervention. MCAv was assessed while seated to examine the posture of interest, sitting, and to prevent the possible confounding haemodynamic effects of moving to a supine posture. Participants then returned to a supine posture and all other measurements were repeated (POST) (Fig. 1).

\section{Study procedures}

\section{Visit 1: familiarisation visit}

Participants completed the battery of cognitive performance tests that would be used during the experimental visit to reduce any learning effects. Participants were given the opportunity to ask questions to ensure full comprehension. In preparation for the experimental visit the following day, participants were provided a standardised breakfast meal (50 $\mathrm{g}$ of porridge oats prepared with water and a banana) to take away with them and were instructed to consume this meal $2 \mathrm{~h}$ prior to their scheduled arrival time, on the morning of their second visit (Fig. 1).

\section{Visit 2: experimental visit}

Prior to the experimental visit, participants were instructed to avoid strenuous exercise for $24 \mathrm{~h}$, and to abstain from caffeine and alcohol. Women were assessed in the follicular phase of the menstrual cycle (days 1-7) to control for hormonal variations influencing cerebrovascular responses [19]. In the $2 \mathrm{~h}$ 


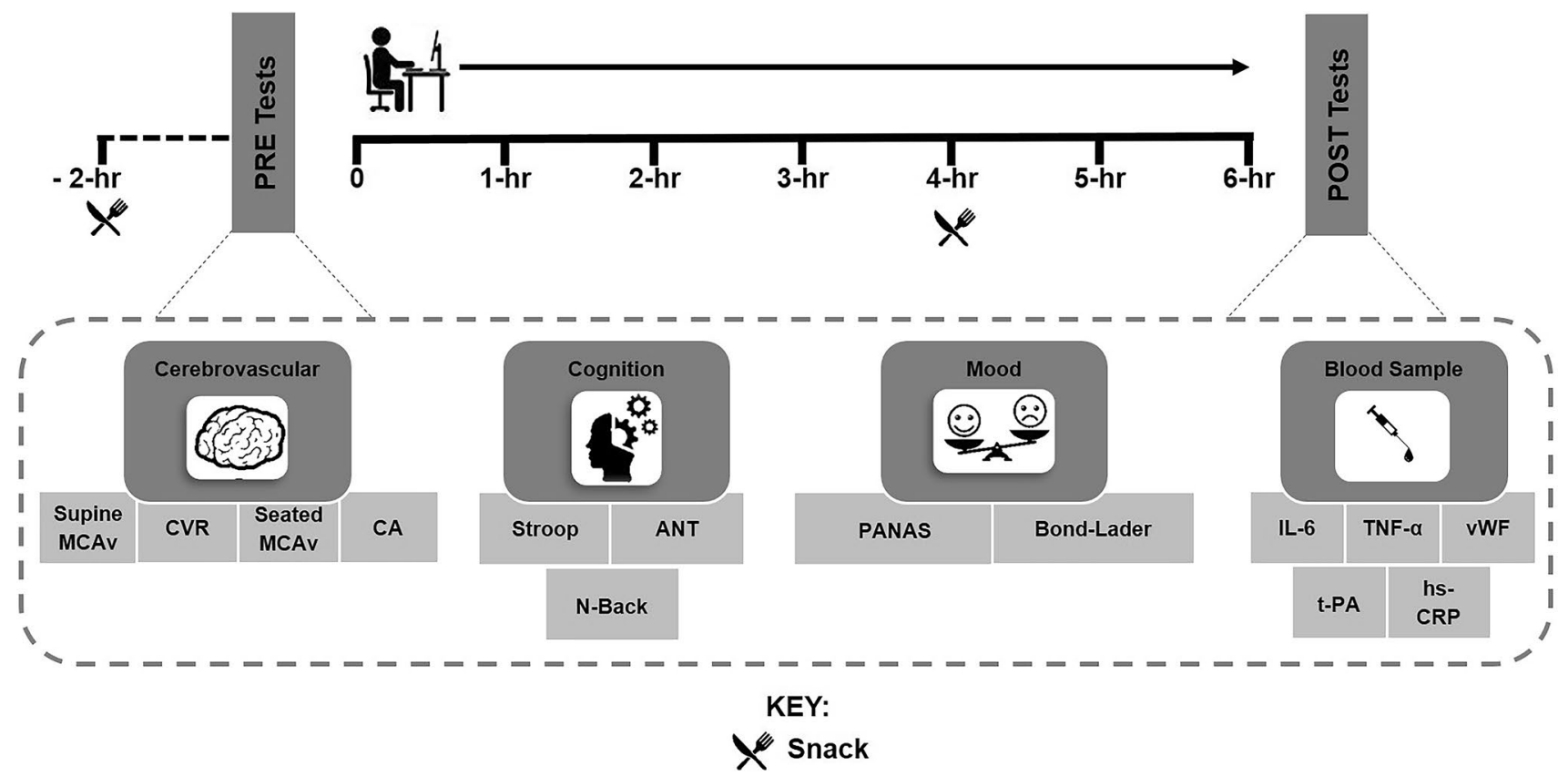

Fig. 1 Study design for the experimental visit. $M C A v$ middle cerebral artery blood flow velocity, $C V R$ cerebrovascular carbon dioxide reactivity, $C A$ cerebral autoregulation, ANT attention network test, PANAS positive and negative affect schedule, IL-6 interleukin-6, TNF- $\alpha$ tumour necrosis factor-alpha, $v W F$ von Willebrand factor, $t-P A$ tissue plasminogen activator, $h s$ - $C R P$ high-sensitivity c-reactive protein between participants consuming the standardised meal and arriving at the laboratory, participants were asked to keep physical activity to a minimum aside from walking to the laboratory. On arrival, participants completed the Workforce Sitting Questionnaire (WSQ) to assess sitting time on a working day and a non-working day [20]. Participants were asked to verbally confirm they had consumed the standardised meal prior to arrival and the time at which this occurred. Participants were given this same meal $2 \mathrm{~h}$ before POST-measurements were taken, ensuring the time between food consumption and physiological measurements were matched between PRE- and POST-assessments (Fig. 1). Water was available to drink ad libitum. During the $6 \mathrm{~h}$ uninterrupted sitting period, participants remained seated at a desk and were permitted to perform low cognitively demanding desk-based activities, such as reading and watching television. Participants were prevented from standing, walking or making vigorous movements during this period, limb movements were otherwise uncontrolled (i.e. fidgeting was permitted). Participants were wheeled to the bathroom if needed (on average participants visited the bathroom once). Participants were continuously supervised to ensure these conditions were adhered to.

\section{Measurements}

\section{Blood sampling}

Blood samples were obtained from the antecubital vein of the forearm via standard venepuncture technique (Vacutainers Systems, Becton-Dickinson). Samples were collected into vacutainers containing Silica (Clot Activator) and stored on ice until centrifugation for 10-min at 1,200 $\mathrm{g}$ at $4{ }^{\circ} \mathrm{C}$. Serum aliquots were stored at $-80{ }^{\circ} \mathrm{C}$ for subsequent analyses. Commercially available pre-coated high-sensitivity ELISA kits (Thermo Fisher Scientific) were used to determine interleukin-6 (IL-6), tumour necrosis factor-alpha (TNF- $\alpha$ ) and high-sensitivity c-reactive protein (hs-CRP), while commercially available pre-coated standard ELISA kits (Thermo Fisher Scientific) were used to determine tissue plasminogen activator (t-PA) and von Willebrand factor (vWF). IL-6, TNF- $\alpha$ and hs-CRP were analysed as they are implicated in mood disorders [21] and cognitive functioning [22]. t-PA and vWF were analysed owing to their association with reductions in CBF [23]. Kits were stored and utilised according to the manufacturer's instructions. An automated 
plate reader (CLARIOstar, BMG LABTECH GmbH, Offenburg, Germany) was used to read the raw absorbance values at the $450 \mathrm{~nm}$ wavelength for all assays. Each sample was analysed in duplicate.

\section{Middle cerebral artery blood flow velocity (MCAv)}

MCAv was used as a surrogate measure for $\mathrm{CBF}$ as the MCA accounts for $70-80 \%$ of the brain's total perfusion [24]. MCAv was assessed using continuous bilateral transcranial Doppler ultrasound (TCD) (ST3, Spencer Technologies, Redmond, WA, USA), with 2-MHz Doppler probes positioned over each temporal window, located above the zygomatic arch and secured using an adjustable headband (Marc 600 Headframe, Spencer Technologies). Each MCA was identified based on the signal depth, peak and mean blood velocity as previously described [15]. Once optimal signals had been obtained, the transducers were secured into position to allow measures to be collected. Probes were removed between PRE- and POST-measures. Signal parameters and photographs of the probe positions were recorded to ensure within-subject consistency between measurements. The sonographer had a between-day coefficient of variation of $7.8 \%$ for MCAv. Supine and seated MCAv were acquired for a 5-min period and mean MCAv calculated from the envelope of the velocity tracing using a weighted mean $(1 / 3$ maximum $+2 / 3$ minimum) to account for the relative time spent in systolic and diastolic pressures [24]. Cerebrovascular conductance (CVC) was calculated by dividing MCAv by mean arterial pressure (MAP).

\section{Cerebrovascular carbon dioxide reactivity (CVR)}

Maintenance of adequate CBF is influenced by the brain's ability to alter blood flow in response to changes in partial pressure of arterial carbon dioxide $\left(\mathrm{CO}_{2}\right)$, termed CVR [25]. Testing procedures have been described in detail elsewhere [17], but briefly, participants were in a supine position and instrumented with a mouth piece (MLA1026, ADInstruments, Colorado Springs, Colorado, USA) with a two-way non-rebreathing valve (MLA1028). After a 1-min baseline, participants voluntarily hyperventilated until the pressure of end tidal $\mathrm{CO}_{2}\left(\mathrm{PETCO}_{2}\right)$ was reduced to $20 \mathrm{mmHg}$. Participants then returned their respiratory rate to normal and inhaled a $5 \% \mathrm{CO}_{2}$ mixture for 3-min. MCAv was continually assessed. Simultaneously, to assess extracranial artery reactivity, left common carotid artery (CCA) arterial diameter and blood flow were measured using a $10-\mathrm{MHz}$ multifrequency linear array probe, attached to high resolution ultrasound machine (uSmart 3300; Terason, Burlington, MA, USA). Absolute and relative MCAv, CCA diameter and CCA blood flow reactivity to the changes in $\mathrm{CO}_{2}$ were calculated as previously described $[17,24]$.

\section{Cerebral autoregulation (CA)}

CA maintains CBF over a range of perfusion pressures [15]. To assess dynamic CA, participants completed a squat-stand test, involving repeated cycles of 5-s standing and 5-s squatting for 5-min to induce oscillations in blood pressure (BP). MCAv and BP were continuously assessed. Data were processed and analysed using standardised transfer function analysis (TFA) guidelines to produce values of gain, normalised gain, phase and coherence for each of the three frequency domains: very low frequency (VLF: $0.02-0.07 \mathrm{~Hz}$ ), low frequency (LF: $0.07-0.2 \mathrm{~Hz}$ ) and high frequency (HF: $0.2-0.5 \mathrm{~Hz}$ ) [26]. These parameters are described in detail elsewhere [17].

\section{Hemodynamics}

Participants were fitted with a photoplethysmographic cuff on the index or middle finger of the right hand (Finometer model 1, Finapres Medical Systems BV, Amsterdam, The Netherlands) and a 3-lead electrocardiogram to continuously assess MAP and heart rate (HR), respectively, throughout measurements.

\section{Mood}

Mood was assessed using two questionnaires: The Positive and Negative Affect Schedule (PANAS) [27] and the BondLader Mood Rating Scale [28]. The PANAS required participants to rate on a scale of 1-5 the extent to which they felt 10 positive and 10 negative states, and is a reliable measure of affect for moment in time assessments [27]. The BondLader Mood Rating Scale included 12 visual analogue scales featuring bipolar end-points for different mood dimensions. These scales were combined to form three mood factors: alert, calm and contented [28].

\section{Cognition}

A battery of computer-based cognitive performance tests was completed using E-Prime software (Version 2.0 Professional, Psychology Software Tools, Pittsburgh, PA). Tests were completed in a randomised order between participants but not within an experimental visit. Three cognitive components were assessed: (1) Executive function using the Stroop Colour-Word test [29] which generated an interference score based on the reaction times from congruent and incongruent stimuli; (2) Attention using the Attention Network Test (ANT) which examined three attentional networks: alerting, orientating and executive control [30]; (3) Working memory using the N-Back Task 
[31] which calculated the response accuracy and time taken to identify whether a presented letter was the same as that presented one, two or three times prior in a letter sequence.

\section{Statistical analyses}

Data were analysed using statistical software (SPSS Version 23.0, IBM Corporation, Somers, NY, USA), with significance accepted as $p<0.05$. Results are presented as means \pm standard deviation (SD). Data were assessed for normal distribution using Shapiro-Wilk tests. Differences between PRE- and POST-data were compared using paired-samples $t$ tests (parametric data) or Wilcoxon signed ranked tests (non-parametric data), with post hoc analyses performed using the least significant difference (LSD) method. Effect size (Cohen's $d$ ) of all significant differences was calculated by dividing the difference in group means by the standard deviation of the pooled data. These were interpreted as: $d=0.2$ considered small, $d=0.5$ considered medium, and $d=0.8$ considered large [32]. Where significant changes were observed in our data following sitting, Pearson's bivariate correlation analysis (parametric data) or Spearman's correlation (non-parametric data) were used to assess the relationship between the changes (POST - PRE) in these outcomes.

\section{Results}

All 25 participants completed the study and were included in analyses. Participants self-reported sitting for $12.1 \pm 3.3 \mathrm{~h}$ during workdays and $10.0 \pm 3.3 \mathrm{~h}$ during

Table 1 Descriptive characteristics and self-reported sitting time of participants $(n=25)$

\begin{tabular}{ll}
\hline & $\begin{array}{l}\text { Mean } \pm \text { SD } \\
\text { or } n \text { of } \\
\text { group }\end{array}$ \\
\hline Age (years) & $28.3 \pm 7.5$ \\
Body Mass $(\mathrm{kg})$ & $74.3 \pm 12.4$ \\
Height $(\mathrm{cm})$ & $175.0 \pm 7.0$ \\
Body mass index $\left(\mathrm{kg} \cdot \mathrm{m}^{-2}\right.$ ) & $24.2 \pm 3.3$ \\
Sitting time per workday (Hours) & $12.1 \pm 3.3$ \\
Sitting time per non-workday (Hours) & $10.0 \pm 3.3$ \\
Job category & \\
Manager/Director & 1 \\
Clerical/Services/Sales & 3 \\
Research/Technical & 21 \\
\hline
\end{tabular}

non-workdays. Full descriptive characteristics are shown in Table 1.

\section{Inflammation}

Following sitting, significant decreases in t-PA (PRE: $3288.3 \pm 2519.5 \mathrm{pg} / \mathrm{ml}$, POST: $2927.9 \pm 2153.8 \mathrm{pg} / \mathrm{ml}$, $p=0.039, d=0.16)$ and hs-CRP (PRE: $1.1 \pm 1.3 \mu \mathrm{g} / \mathrm{ml}$, POST: $0.1 \pm 1.1 \mu \mathrm{g} / \mathrm{ml}, p=0.028, d=0.85)$ were observed. There were no significant changes in IL-6 (PRE: $1.9 \pm 2.1 \mathrm{pg} /$ $\mathrm{ml}$, POST: $1.5 \pm 1.7 \mathrm{pg} / \mathrm{ml}, p=0.170)$, TNF- $\alpha$ (PRE: $1.3 \pm 2.0 \mathrm{pg} / \mathrm{ml}$, POST: $0.6 \pm 1.0 \mathrm{pg} / \mathrm{ml}, p=0.107)$, or vWF (PRE: $6571.6 \pm 5227.2 \mathrm{ng} / \mathrm{ml}$, POST: $6173.0 \pm 3842.6 \mathrm{ng} /$ $\mathrm{ml}, p=0.313)$.

\section{Cardiorespiratory and haemodynamic measures}

HR in the supine $(p=0.022, d=0.48)$ and seated $(p=0.003$, $d=0.49$ ) postures were significantly reduced at POST compared to PRE (Table 2). There was also a significant reduction in seated MAP ( $p=0.001, d=0.56)$ between PRE and POST, but not for supine MAP ( $p=0.966$; Table 2). There was no significant difference between PRE and POST supine $(p=0.365)$ or seated $(p=0.306) \mathrm{PETCO}_{2}$ (Table 2$)$.

\section{Middle cerebral artery blood flow velocity}

Seated MCAv significantly reduced following sitting (PRE: $58.2 \pm 7.3 \mathrm{~cm} \cdot \mathrm{s}^{-1}$, POST: $54.8 \pm 7.1 \mathrm{~cm} \cdot \mathrm{s}^{-1}, p=0.001$, $d=0.48$ ); however, there was no significant change in seated CVC (PRE: $0.65 \pm 0.12 \mathrm{~cm} \cdot \mathrm{s}^{-1} \cdot \mathrm{mmHg}^{-1}$, POST: $0.65 \pm 0.11 \mathrm{~cm} \cdot \mathrm{s}^{-1} \cdot \mathrm{mmHg}^{-1}, p=0.950 ;$ Fig. 2a). In the supine posture, significant reductions in MCAv (PRE: $63.5 \pm 8.1 \mathrm{~cm} \cdot \mathrm{s}^{-1}$, POST: $60.6 \pm 9.0 \mathrm{~cm} \cdot \mathrm{s}^{-1}, p=0.012$, $d=0.35$ ) and CVC (PRE: $0.77 \pm 0.15 \mathrm{~cm} \cdot \mathrm{s}^{-1} \cdot \mathrm{mmHg}^{-1}$,

Table 2 Cardiorespiratory measures prior to (PRE) and following (POST) $6 \mathrm{~h}$ of uninterrupted sitting (mean $\pm \mathrm{SD}$ )

\begin{tabular}{lccc}
\hline & \multicolumn{1}{l}{ PRE } & \multicolumn{1}{l}{ POST } & $p$-value \\
\hline Supine position & & & \\
MAP (mmHg) & $84 \pm 8$ & $84 \pm 8$ & 0.966 \\
HR (bpm) & $62 \pm 12$ & $57 \pm 9^{*}$ & 0.022 \\
PETCO $(\mathrm{mmHg})$ & $38.4 \pm 2.9$ & $38.8 \pm 3.6$ & 0.365 \\
Seated position & & & \\
MAP (mmHg) & $90 \pm 10$ & $85 \pm 8^{*}$ & 0.001 \\
HR (bpm) & $66 \pm 10$ & $61 \pm 11^{*}$ & 0.003 \\
PETCO $_{2}(\mathrm{mmHg})$ & $36.6 \pm 3.0$ & $36.8 \pm 3.9$ & 0.306 \\
\hline
\end{tabular}

$M A P$ mean arterial pressure, $\mathrm{HR}$ heart rate, $\mathrm{PETCO}_{2}$ pressure of endtidal carbon dioxide

*Significantly different to PRE $(p<0.05)$ 

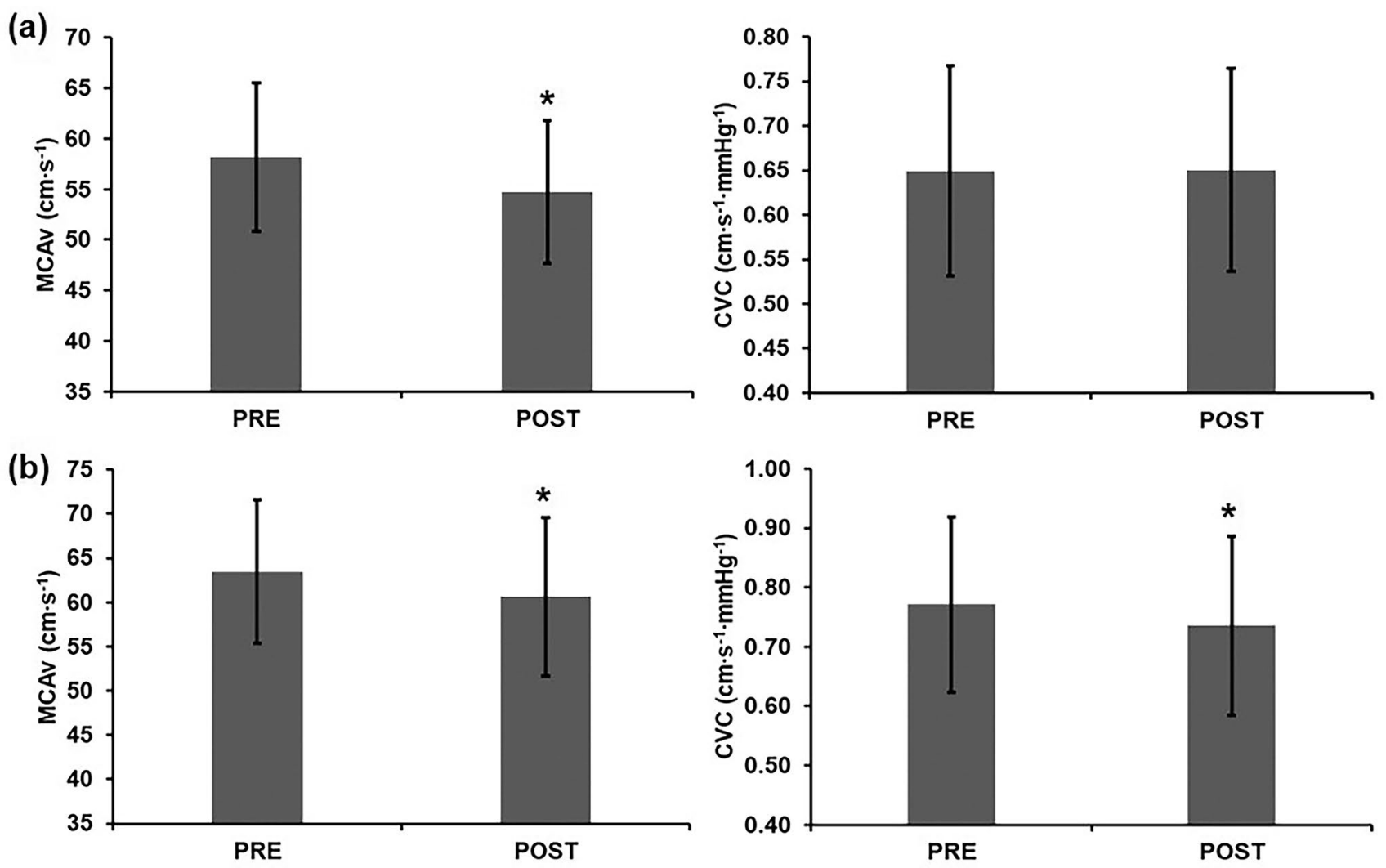

Fig. 2 Middle cerebral artery blood flow velocity (MCAv) and cerebrovascular conductance (CVC) in a seated and $\mathbf{b}$ supine postures prior to (PRE) and following (POST) $6 \mathrm{~h}$ of uninterrupted sitting. Error bars $= \pm$ SD. *Significantly different to PRE $(p<0.05)$

POST: $0.74 \pm 0.15 \mathrm{~cm} \cdot \mathrm{s}^{-1} \cdot \mathrm{mmHg}^{-1}, p=0.018, d=0.15$ ) were observed (Fig. 2b).

\section{Cerebrovascular carbon dioxide reactivity}

No significant differences were observed between absolute or relative MCA CVR or CCA CVR ( $p>0.05$; Table 3$)$.

\section{Cerebral autoregulation}

In the VLF, there was a significant increase in normalised gain following uninterrupted sitting ( $p=0.016, d=0.94$; Table 3 ). There were no significant changes for any other parameters in any of the frequency domains $(p>0.05)$.

\section{Mood}

There were significant decreases in positive affect $(\mathrm{p}<0.001$, $d=0.62)$, and the alert $(p=0.002, d=0.64)$ and contented $(p=0.006, d=0.52)$ mood states (Table 4$)$. Negative affect $(p=0.610)$ and the calm mood state $(p=0.392)$ did not significantly change.

\section{Cognition}

Following uninterrupted sitting, there were no significant changes in any measures of cognition ( $p>0.05$; Table 4$)$.

\section{Relationship between changes in inflammation, cerebrovascular function and mood}

There were no significant relationships between the changes in t-PA and hs-CRP and the changes in mood $(p>0.05)$. The changes in seated or supine MCAv and VLF normalised gain were also not significantly associated with the changes in $\operatorname{mood}(p>0.05)$.

\section{Discussion}

This study explored whether sitting-induced alterations in inflammation and cerebrovascular function are related to changes in mood and cognition. We observed that sitting acutely decreased levels of hs-CRP and t-PA inflammatory markers, reduced MCAv, impaired dynamic CA and decreased aspects of mood; but had no influence on cognition. The observed changes in inflammation and 
Table 3 Values of cerebral autoregulation (CA) and cerebrovascular carbon dioxide reactivity $(\mathrm{CVR})$ before (PRE) and after (POST) $6 \mathrm{~h}$ of uninterrupted sitting $($ mean $\pm \mathrm{SD})$

\begin{tabular}{|c|c|c|c|}
\hline & PRE & POST & $p$-value \\
\hline \multicolumn{4}{|l|}{ MCA CVR } \\
\hline $\operatorname{CVR}\left(\mathrm{R}^{2}\right)$ & $0.84 \pm 0.08$ & $0.84 \pm 0.09$ & - \\
\hline Absolute MCAv $\left(\mathrm{cm} \cdot \mathrm{s}^{-1} \cdot \mathrm{mmHg}^{-1}\right)$ & $3.12 \pm 0.65$ & $3.12 \pm 0.79$ & 0.992 \\
\hline Relative MCAv $\left(\% \cdot \mathrm{mmHg}^{-1}\right)$ & $4.82 \pm 0.86$ & $4.13 \pm 1.23$ & 0.198 \\
\hline \multicolumn{4}{|l|}{ CCA CVR } \\
\hline Absolute CCA Diameter $\left(\mathrm{cm} \cdot \mathrm{mmHg}^{-1}\right)$ & $0.002 \pm 0.002$ & $0.003 \pm 0.003$ & 0.235 \\
\hline Relative CCA Diameter $\left(\% \cdot \mathrm{mmHg}^{-1}\right)$ & $0.33 \pm 0.28$ & $0.39 \pm 0.38$ & 0.904 \\
\hline Absolute CCA Blood Flow $\left(\mathrm{ml} \cdot \mathrm{min}^{-1} \cdot \mathrm{mmHg}^{-1}\right)$ & $0.32 \pm 0.31$ & $0.41 \pm 0.25$ & 0.164 \\
\hline Relative CCA Blood Flow $\left(\% \cdot \mathrm{mmHg}^{-1}\right)$ & $2.42 \pm 2.17$ & $3.18 \pm 1.99$ & 0.122 \\
\hline \multicolumn{4}{|l|}{ CA: VLF } \\
\hline Phase (degrees) & $41.0 \pm 16.7$ & $42.1 \pm 13.9$ & 0.857 \\
\hline Gain $\left(\mathrm{cm} \cdot \mathrm{s}^{-1} \cdot \mathrm{mmHg}^{-1}\right)$ & $0.50 \pm 0.13$ & $0.65 \pm 0.17$ & 0.051 \\
\hline $\operatorname{Gain}_{\mathrm{n}}\left(\% \cdot \mathrm{mmHg}^{-1}\right)$ & $0.86 \pm 0.20$ & $1.09 \pm 0.29 *$ & 0.016 \\
\hline Coherence & $0.5 \pm 0.08$ & $0.5 \pm 0.11$ & - \\
\hline \multicolumn{4}{|l|}{ CA: LF } \\
\hline Phase (degrees) & $24.4 \pm 18.5$ & $23.0 \pm 13.3$ & 0.759 \\
\hline Gain $\left(\mathrm{cm} \cdot \mathrm{s}^{-1} \cdot \mathrm{mmHg}^{-1}\right)$ & $0.79 \pm 0.18$ & $0.85 \pm 0.23$ & 0.282 \\
\hline $\operatorname{Gain}_{\mathrm{n}}\left(\% \cdot \mathrm{mmHg}^{-1}\right)$ & $1.28 \pm 0.25$ & $1.39 \pm 0.33$ & 0.153 \\
\hline Coherence $^{\#}$ & $0.6 \pm 0.11$ & $0.6 \pm 0.11$ & - \\
\hline \multicolumn{4}{|l|}{ CA: HF } \\
\hline Phase (degrees) & $10.6 \pm 34.8$ & $12.4 \pm 21.2$ & 0.785 \\
\hline Gain $\left(\mathrm{cm} \cdot \mathrm{s}^{-1} \cdot \mathrm{mmHg}^{-1}\right)$ & $0.83 \pm 0.34$ & $0.80 \pm 0.27$ & 0.727 \\
\hline $\operatorname{Gain}_{\mathrm{n}}\left(\% \cdot \mathrm{mmHg}^{-1}\right)$ & $1.34 \pm 0.51$ & $1.32 \pm 0.39$ & 0.875 \\
\hline Coherence $^{\#}$ & $0.4 \pm 0.12$ & $0.4 \pm 0.13$ & - \\
\hline
\end{tabular}

$M C A$ middle cerebral artery, $C V R$ cerebrovascular carbon dioxide reactivity, $M C A v$ middle cerebral artery blood flow velocity, $C C A$ common carotid artery, $C A$ cerebral autoregulation, $V L F$ very low frequency, $L F$ low frequency, $H F$ high frequency, Gain $_{n}$ normalised gain

\#Coherence values were used to accept the validity of gain and phase estimates and not statistically compared

*Significantly different to PRE $(p<0.05)$ cerebrovascular function were not related to the changes in mood. Overall, our results indicate that neither inflammation nor cerebrovascular function are associated with this lowered mood state, suggesting other mechanisms may underlie the changes in mood in response to acute sitting.

Markers of inflammation decreased following an acute, prolonged sitting period, in contrast to our hypothesis and previous research observing increases in salivary IL- 8 following an acute sitting bout [13]. Methodological differences between salivary and venous measures of inflammation could explain this disparity [33, 34]. Additionally, the controlled testing environment and completion of low cognitively demanding activities while seated may have removed participants from the daily stresses of their typical life, causing inflammatory markers to decrease. In support, acute stress activates peripheral inflammatory pathways [35], thus the removal of such stressors could have the opposite effect. This raises important methodological consideration for future experimental work assessing acute sitting and mood.
In support of previous research [17], acute sitting impaired MCAv and dynamic CA, aspects of cerebrovascular function. Importantly, the decline in MCAv (marker of $\mathrm{CBF}$ ) following sitting is unlikely due to daily circadian variation of CBF. CBF closely tracks the rhythm of core body temperature and is therefore lower during the morning than in the afternoon or evening [36]. Since our data shows a reduction in MCAv from baseline (am) to post test (pm), this decline most likely relates to prolonged sitting rather than a circadian rhythm. Although the mechanisms of CA are not fully elucidated, it is suggested that sympathetic activity, endothelial nitric oxide production and myogenic factors all contribute [37]. In peripheral vessels, sitting-induced impairment in vascular function is likely in part due to reduced nitric oxide production and heightened sympathetic activity [38]. Similar mechanisms may therefore contribute to the impaired CA observed in this study.

Prolonged sitting negatively impacted aspects of mood, supporting previous observations that reducing sitting acutely improves mood $[6,7]$. Heightened inflammation may 
Table 4 Mood and cognitive performance outcomes prior to (PRE) and after (POST) $6 \mathrm{~h}$ of uninterrupted sitting (mean \pm SD)

\begin{tabular}{|c|c|c|c|}
\hline & PRE & POST & $p$-value \\
\hline \multicolumn{4}{|l|}{ Mood } \\
\hline Positive affect & $27.1 \pm 7.2$ & $22.5 \pm 7.9^{*}$ & $<0.001$ \\
\hline Negative affect & $12.5 \pm 3.3$ & $12.1 \pm 2.3$ & 0.610 \\
\hline Alert & $53.6 \pm 15.0$ & $43.0 \pm 18.5^{*}$ & 0.002 \\
\hline Calm & $48.2 \pm 10.6$ & $45.9 \pm 8.9$ & 0.392 \\
\hline Content & $67.7 \pm 13.7$ & $60.3 \pm 15.5^{*}$ & 0.006 \\
\hline \multicolumn{4}{|c|}{ Executive function (Stroop Colour-Word Test) } \\
\hline Interference score (ms) & $185 \pm 120$ & $171 \pm 133$ & 0.425 \\
\hline \multicolumn{4}{|c|}{ Attention (Attention Network Test) } \\
\hline Alerting network (ms) & $13 \pm 21$ & $15 \pm 17$ & 0.638 \\
\hline Orientating network (ms) & $15 \pm 28$ & $18 \pm 18$ & 0.584 \\
\hline Executive control (ms) & $73 \pm 22$ & $75 \pm 24$ & 0.647 \\
\hline \multicolumn{4}{|c|}{ Working memory (N-Back Task) } \\
\hline One back accuracy $(\%)$ & $93.8 \pm 8.6$ & $93.0 \pm 6.0$ & 0.192 \\
\hline One back RT (ms) & $599 \pm 121$ & $605 \pm 169$ & 0.737 \\
\hline Two back Accuracy (\%) & $92.8 \pm 10.3$ & $85.6 \pm 21.3$ & 0.153 \\
\hline Two back RT (ms) & $877 \pm 349$ & $837 \pm 294$ & 0.437 \\
\hline Three back accuracy (\%) & $80.8 \pm 17.2$ & $75.8 \pm 20.7$ & 0.112 \\
\hline Three back RT (ms) & $1326 \pm 811$ & $1377 \pm 987$ & 0.586 \\
\hline
\end{tabular}

$R T$ reaction time

*Significantly different to PRE $(p<0.05)$

contribute to sitting-induced decreases in mood [14]; however, inflammation decreased in our study. Consequently, it was unsurprising that the changes in mood were not associated with any inflammatory markers as there would be no underlying physiological reason for a relationship between these variables. Furthermore, the decreases in mood were not correlated with the reductions in MCAv or CA we observed, indicating cerebrovascular function may not be a mechanism explaining acute mood alterations. An alternative mechanism relating to acute, prolonged sitting may therefore explain our mood data. Indeed, the reduction in mood may relate to the laboratory testing setting and participant boredom, which cannot be ruled out as potential contributing factors.

No changes in cognition were observed following sitting, which contrasts previous research in older adults [9] and Qatari females [8]. Differences between our findings may relate to the variety of tests used to assess each cognitive domain, in addition to the populations assessed, since other studies have observed no influence on cognition following acute sitting bouts when assessing younger, adult males and females $[6,39]$. It has been hypothesised that hypoperfusion of the brain due to prolonged sitting contributes to cognitive decline [40], but despite decreases in MCAv observed in this study, this did not translate to changes in cognition, indicating that in an acute setting changes in MCAv are not associated with cognition. However, MCAv does not measure regional blood flow and it has been recently observed that prefrontal cortex perfusion was unaltered following an acute sitting bout and executive function was also unchanged [41]. This suggests that acutely, whilst MCAv decreases, perfusion and oxygen delivery to specific brain regions is maintained, which may preserve cognition. Instead, chronic exposure to sitting-induced decreases in MCAv may cause structural and function damage, leading to long-term cognitive impairment. Future research should explore these mechanisms in more detail.

\section{Limitations}

The cognitive tests selected may not have been sensitive enough to detect an effect of sitting, or we may have assessed domains of cognition that are not influenced by sitting. A learning effect may have also occurred for the cognitive tests, although the inclusion of a familiarisation visit aimed to reduce this risk. The desk-based activities participants completed during sitting were not controlled, therefore they may have differentially influenced cerebrovascular, cognitive and mood responses. Due to the length of the experimental protocol, measurements could not be completed in a fasted state as is usual best practice. However, the timing and content of the meals prior to each measurement time-point were matched, so any postprandial influence on outcomes measures would be similar.

\section{Conclusion}

This study demonstrates that in healthy desk workers, alterations in inflammation or cerebrovascular function following $6 \mathrm{~h}$ of prolonged, uninterrupted sitting are not related to the observed reductions in mood. Overall, this study provides initial, exploratory data that future experimental research should investigate further to determine the mechanisms underlying the influence of sitting on mood and cognition.

Author contributions SC, NH, DT, and RD contributed to the conception and design of the study. SC completed data collection. CS and AM completed ELISA analyses. SC analysed all other data. SC and $\mathrm{NH}$ interpreted the data and drafted the initial manuscript. All authors contributed to the critical revision of the manuscript, approve the final submission and take responsibility for the integrity of the data and the accuracy of the data analysis.

Funding This work was funded by a Biotechnology and Biological Sciences Research Council (BBSRC) Industrial CASE research Grant (BB/L017237/1) in collaboration with Unilever. 
Availability of data and materials The datasets used and/or analysed during the current study are available from the corresponding author on reasonable request.

\section{Declarations}

Conflict of interests SC received $\mathrm{PhD}$ scholarship funding from a Biotechnology and Biological Sciences Research Council (BBSRC) grant. $\mathrm{RD}$ is employed by Unilever, which has commercial interests in Food, Home and Personal Care products. All other authors declare they have no conflict of interest. The BBSRC had no role in the design and conduct of the study; collection, management, analysis, and interpretation of the data; and preparation, review, or approval of this manuscript.

Ethics approval Study procedures were approved by the Liverpool John Moores University Ethics Committee (16/SPS/031) and conformed to the Declaration of Helsinki.

Consent to participate All participants provided written informed consent prior to inclusion.

Open Access This article is licensed under a Creative Commons Attribution 4.0 International License, which permits use, sharing, adaptation, distribution and reproduction in any medium or format, as long as you give appropriate credit to the original author(s) and the source, provide a link to the Creative Commons licence, and indicate if changes were made. The images or other third party material in this article are included in the article's Creative Commons licence, unless indicated otherwise in a credit line to the material. If material is not included in the article's Creative Commons licence and your intended use is not permitted by statutory regulation or exceeds the permitted use, you will need to obtain permission directly from the copyright holder. To view a copy of this licence, visit http://creativecommons.org/licenses/by/4.0/.

\section{References}

1. Tremblay MS, Aubert S, Barnes JD et al (2017) Sedentary behavior research network (SBRN)-terminology consensus project process and outcome. Int J Behav Nutr Phys Act 14:75. https:// doi.org/10.1186/s12966-017-0525-8

2. Zhai L, Zhang Y, Zhang D (2015) Sedentary behaviour and the risk of depression: a meta-analysis. Br J Sports Med 49:705-709. https://doi.org/10.1136/bjsports-2014-093613

3. Teychenne M, Costigan SA, Parker K (2015) The association between sedentary behaviour and risk of anxiety: a systematic review. BMC Public Health 15:513. https://doi.org/10.1186/ s12889-015-1843-x

4. Edwards MK, Loprinzi PD (2017) Combined associations of sedentary behavior and cardiorespiratory fitness on cognitive function among older adults. Int J Cardiol 229:71-74. https://doi.org/10. 1016/j.ijcard.2016.11.264

5. Bakrania K, Edwardson C, Khunti K et al (2018) Associations between sedentary behaviours and cognitive function: crosssectional and prospective findings from the UK Biobank. Am J Epidemiol 187:441-454. https://doi.org/10.1093/aje/kwx273

6. Bergouignan A, Legget KT, De Jong N et al (2016) Effect of frequent interruptions of prolonged sitting on self-perceived levels of energy, mood, food cravings and cognitive function. Int J Behav Nutr Phys Act 13:113. https://doi.org/10.1186/s12966-016-0437-z

7. Sperlich B, De Clerck I, Zinner C et al (2018) Prolonged sitting interrupted by 6-min of high-intensity exercise: circulatory, metabolic, hormonal, thermal, cognitive, and perceptual responses. Front Physiol. https://doi.org/10.3389/fphys.2018. 01279

8. Chrismas BCR, Taylor L, Cherif A et al (2019) Breaking up prolonged sitting with moderate-intensity walking improves attention and executive function in Qatari females. PLoS ONE 14:1-13. https://doi.org/10.1371/journal.pone.0219565

9. Wheeler MJ, Green DJ, Ellis KA et al (2020) Distinct effects of acute exercise and breaks in sitting on working memory and executive function in older adults: a three-arm, randomised cross-over trial to evaluate the effects of exercise with and without breaks in sitting on cognition. Br J Sports Med 54:776-781. https://doi.org/ 10.1136/bjsports-2018-100168

10. Clemes SA, Houdmont J, Munir F et al (2016) Descriptive epidemiology of domain-specific sitting in working adults: the Stormont Study. J Public Health (Bangkok) 38:53-60. https:// doi.org/10.1093/pubmed/fdu114

11. Rosenblat JD, Cha DS, Mansur RB, McIntyre RS (2014) Inflamed moods: a review of the interactions between inflammation and mood disorders. Prog Neuro-Psychopharmacology Biol Psychiatry 53:23-34. https://doi.org/10.1016/j.pnpbp.2014. 01.013

12. Wright CE, Strike PC, Brydon L, Steptoe A (2005) Acute inflammation and negative mood: mediation by cytokine activation. Brain Behav Immun 19:345-350. https://doi.org/10.1016/j.bbi. 2004.10.003

13. Dogra S, Wolf M, Jeffrey MP et al (2019) Disrupting prolonged sitting reduces IL-8 and lower leg swell in active young adults. BMC Sports Sci Med Rehabil 11:1-7. https://doi.org/10.1186/ s13102-019-0138-4

14. Endrighi R, Steptoe A, Hamer M (2016) The effect of experimentally induced sedentariness on mood and psychobiological responses to mental stress. Br J Psychiatry 208:245-251. https:// doi.org/10.1192/bjp.bp.114.150755

15. Willie CK, Colino FL, Bailey DM et al (2011) Utility of transcranial Doppler ultrasound for the integrative assessment of cerebrovascular function. J Neurosci Methods 196:221-237. https://doi. org/10.1016/j.jneumeth.2011.01.011

16. Wolters FJ, Zonneveld HI, Hofman A et al (2017) Cerebral perfusion and the risk of dementia: a population-based study. Circulation 136:719-728. https://doi.org/10.1161/CIRCULATIONAHA. 117.027448

17. Carter SE, Draijer R, Holder SM et al (2018) Regular walking breaks prevent the decline in cerebral blood flow associated with prolonged sitting. J Appl Physiol 125:790-798. https://doi.org/10. 1152/japplphysiol.00310.2018

18. Ogoh S (2017) Relationship between cognitive function and regulation of cerebral blood flow. J Physiol Sci 67:345-351. https:// doi.org/10.1007/s12576-017-0525-0

19. Peltonen GL, Harrell JW, Aleckson BP et al (2016) Cerebral blood flow regulation in women across menstrual phase: differential contribution of cyclooxygenase to basal, hypoxic, and hypercapnic vascular tone. Am J Physiol Regul Integr Comp Physiol 311:R222-R231. https://doi.org/10.1152/ajpregu.00106.2016

20. Chau JY, van der Ploeg HP, Dunn S et al (2011) A tool for measuring workers' sitting time by domain: the Workforce Sitting Questionnaire. Br J Sports Med 45:1216-1222. https://doi.org/10.1136/ bjsports-2011-090214

21. Felger JC (2017) Imaging the role of inflammation in mood and anxiety-related disorders. Curr Neuropharmacol 15:533-558. https://doi.org/10.2174/1570159x15666171123201142

22. Marsland AL, Gianaros PJ, Kuan DCH et al (2015) Brain morphology links systemic inflammation to cognitive function in midlife adults. Brain Behav Immun 48:195-204. https://doi.org/ 10.1016/j.bbi.2015.03.015 
23. Sabayan B, Westendorp RG, van der Grond J et al (2014) Markers of endothelial dysfunction and cerebral blood flow in older adults. Neurobiol Aging 35:373-377. https://doi.org/10.1016/j.neurobiola ging.2013.08.020

24. Skow RJ, MacKay CM, Tymko MM et al (2013) Differential cerebrovascular $\mathrm{CO} 2$ reactivity in anterior and posterior cerebral circulations. Respir Physiol Neurobiol 189:76-86. https://doi.org/ 10.1016/j.resp.2013.05.036

25. Willie CK, Tzeng Y-C, Fisher JA, Ainslie PN (2014) Integrative regulation of human brain blood flow. J Physiol 592:841-859. https://doi.org/10.1113/jphysiol.2013.268953

26. Claassen JAHR, Meel-van den Abeelen ASS, Simpson DM et al (2016) Transfer function analysis of dynamic cerebral autoregulation: a white paper from the International Cerebral Autoregulation Research Network. J Cereb blood flow Metab 36:665-680. https:// doi.org/10.1177/0271678X15626425

27. Watson D, Clark LA, Tellegen A (1988) Development and validation of brief measures of positive and negative affect: the PANAS scales. J Pers Soc Psychol 54:1063-1070. https://doi.org/10.1037/ 0022-3514.54.6.1063

28. Lader MH, Bond AJ (1998) Interaction of pharmacological and psychological treatments of anxiety. Br J Psychiatry Suppl 47:42 48. https://doi.org/10.1111/j.2044-8341.1974.tb02285.x

29. Stroop JR (1935) Studies of interference in serial verbal reactions. J Exp Psychol 18:643-662. https://doi.org/10.1037/h0054651

30. Fan J, McCandliss BD, Sommer T et al (2002) Testing the efficiency and independence of attentional networks. J Cogn Neurosci 14:340-347. https://doi.org/10.1162/089892902317361886

31. Kirchner WK (1958) Age differences in short-term retention of rapidly changing information. J Exp Psychol 55:352-358. https:// doi.org/10.1037/h0043688

32. Cohen J (1988) Statistical power analysis for the behavioral sciences, 2nd edn. Lawrence Erlbaum Associate, Hillsdale

33. Slavish DC, Graham-Engeland JE, Smyth JM, Engeland CG (2015) Salivary markers of inflammation in response to acute stress. Brain Behav Immun 44:253-269. https://doi.org/10.1016/j. bbi.2014.08.008
34. Williamson S, Munro C, Pickler R et al (2012) Comparison of biomarkers in blood and saliva in healthy adults. Nurs Res Pract 2012:1-4. https://doi.org/10.1155/2012/246178

35. Rohleder N (2019) Stress and inflammation - the need to address the gap in the transition between acute and chronic stress effects. Psychoneuroendocrinology 105:164-171. https://doi.org/10. 1016/j.psyneuen.2019.02.021

36. Conroy DA, Spielman AJ, Scott RQ (2005) Daily rhythm of cerebral blood flow velocity. J Circadian Rhythms 3:3. https://doi. org/10.1186/1740-3391-3-3

37. Tzeng Y-C, Ainslie PN (2014) Blood pressure regulation IX: cerebral autoregulation under blood pressure challenges. Eur J Appl Physiol 114:545-559. https://doi.org/10.1007/s00421-013-2667-y

38. Carter S, Hartman Y, Holder S et al (2017) Sedentary behavior and cardiovascular disease risk: mediating mechanisms. Exerc Sport Sci Rev 45:80-86. https://doi.org/10.1249/JES.0000000000 000106

39. Wennberg P, Boraxbekk C-J, Wheeler M et al (2016) Acute effects of breaking up prolonged sitting on fatigue and cognition: a pilot study. BMJ Open 6:e009630. https://doi.org/10.1136/bmjop en-2015-009630

40. Wheeler MJ, Dempsey PC, Grace MS et al (2017) Sedentary behavior as a risk factor for cognitive decline? A focus on the influence of glycemic control in brain health. Alzheimer's Dement 3:291-300. https://doi.org/10.1016/j.trci.2017.04.001

41. Stoner L, Willey Q, Evans WS et al (2019) Effects of acute prolonged sitting on cerebral perfusion and executive function in young adults: a randomized cross-over trial. Psychophysiology. https://doi.org/10.1111/psyp.13457

Publisher's Note Springer Nature remains neutral with regard to jurisdictional claims in published maps and institutional affiliations. 